\title{
Assessment of coxarthritis risk with dimensionless biomechanical parameters
}

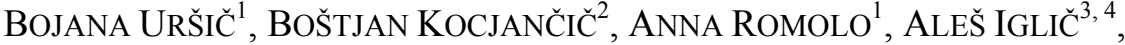 \\ VERONIKA KRALJ-IGLIČ ${ }^{1}{ }^{*}$, OSKAR ZUPANC ${ }^{2}$ \\ ${ }^{1}$ University of Ljubljana, Faculty of Health Sciences, Ljubljana, Slovenia. \\ ${ }^{2}$ University Medical Centre Ljubljana, Department of Orthopaedic Surgery, Ljubljana, Slovenia. \\ ${ }^{3}$ Faculty of Medicine, University of Ljubljana, Ljubljana, Slovenia. \\ ${ }^{4}$ University of Ljubljana, Faculty of Electrical Engineering, Ljubljana, Slovenia.
}

\begin{abstract}
Purpose: Unfavourable distribution of contact stress over the load bearing area is considered a risk factor for early coxarthritis and it is of interest to outline respective biomechanical parameters for its prediction. The purpose of the work was to develop a transparent mathematical model which can be used to assess contact stress in the hip from imaged structures of pelvis and proximal femora, in large population studies and in clinical practice. Methods: We upgraded a previously validated three-dimensional mathematical model of the human hip in the one-legged stance HIPSTRESS by introducing parameters independent from the size of the structures in the images. We validated a new parameter - dimensionless peak stress normalized by the body weight and by the radius of the femoral head $\left(p_{\max } r^{2} / W_{\mathrm{B}}\right)$ on the population of 172 hips that were in the childhood subjected to the Perthes disease and exhibited increased proportion of dysplastic hips. Results: The dimensionless parameter $p_{\max } r^{2} / W_{\mathrm{B}}$ exhibited smaller number of indecisive cases of hip dysplasia predicted by the model than the previously used parameter $p_{\max } / W_{\mathrm{B}}(6 \% \mathrm{vs} .81 \%$, respectively). A threshold for an increased risk of early coxarthritis development by the HIPSTRESS parameter $H=p_{\max } r^{2} / W_{\mathrm{B}}$ was found to be 2 . Conclusions: We proposed a dimensionless peak stress on the load bearing area with the border value of 2 as a decisive parameter over which hips are at risk for early development of degenerative processes and presented a method for determination of biomechanical parameters with the use of nomogram.
\end{abstract}

Key words: hip stress, hip arthritis, hip dysplasia, resultant hip force

\section{Introduction}

Unfavourable contact hip stress has been considered a risk factor for early coxarthrisis development [7], which in turn, affects the morphology and function of hips and pelvis [6]. After the insight into the body became available by X-rays, coxarthrisis development was connected to particularities in hip and pelvis geometry. For example, hips with poor coverage of the femoral head with the acetabulum, hips in which the centre of the head was displaced from the centre of the acetabulum and hips with femoral head contour (of the part under the acetabulum) that deviates from that of the spherical surface, were considered "dysplastic" [21].
The parameter indicating the size of the load-bearing area that can be assessed from the X-ray images of the anteroposterior radiograph or magnetic resonance image is the centre-edge angle of Wiberg $\vartheta_{\mathrm{CE}}$ [24] (Fig. 1A) - the angle between the vertical and the line connecting the centre of the femoral head and the lateral border of the acetabular roof.

Operative procedures have been proposed and performed to prevent or decelerate cartilage degeneration caused by supposedly too high stress. As stress is a mechanical parameter that describes the distribution of load over the load-bearing area, the operative procedures are directed towards augmenting the size of the weight bearing area by increasing the acetabular roof over the femoral head [23].

\footnotetext{
* Corresponding author: Veronika Kralj-Iglič, University of Ljubljana, Faculty of Health Sciences, Zdravstvena pot 5, SI-1000 Ljubljana, Slovenia. Phone +38641720766, e-mail: veronika.kralj-iglic@ffe.uni-lj.si

Received: October 19th, 2020

Accepted for publication: December 10th, 2020
} 


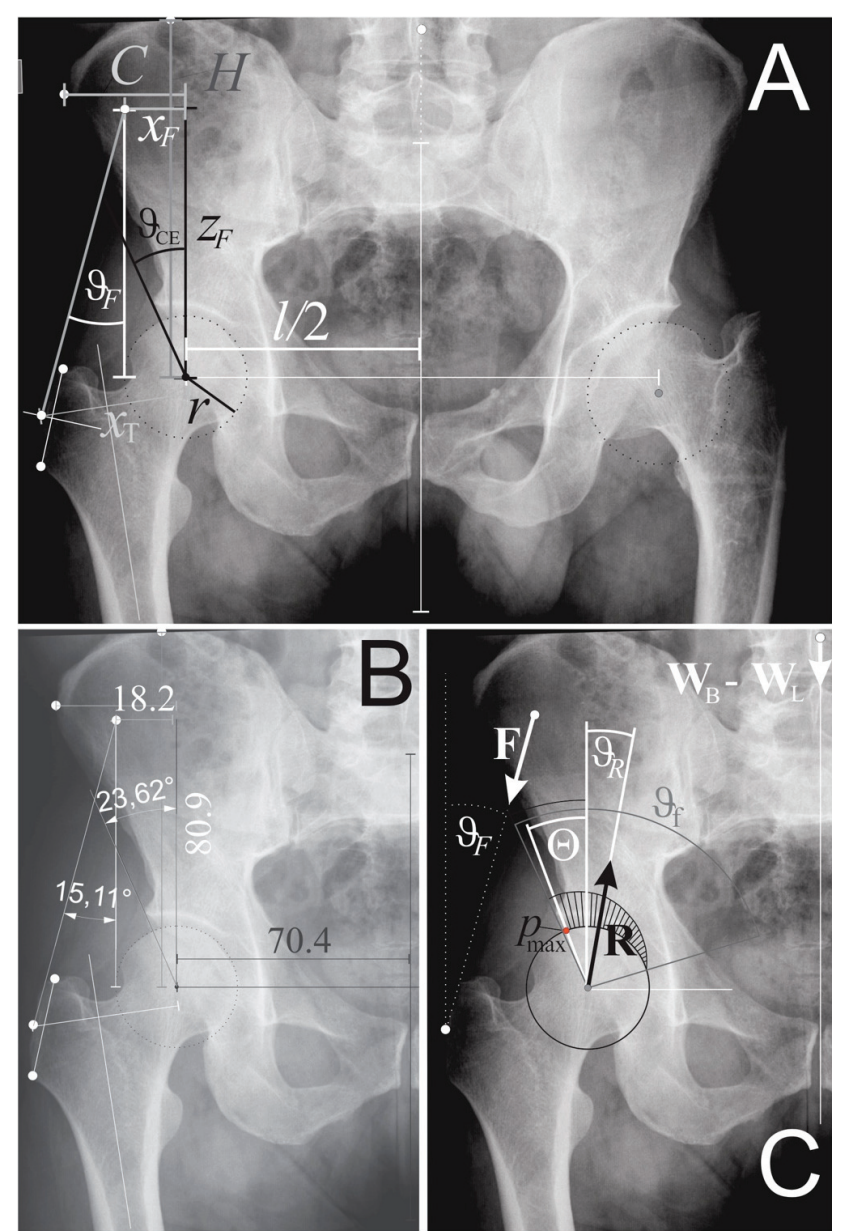

Fig. 1. A) Geometrical parameters of the one-muscle HIPSTRESS model for resultant hip force of an example hip and pelvis;

B) image with measured values of the geometrical parameters. The distances are given in millimeters; C) Biomechanical parameters of the HIPSTRESS model; forces are represented by the vectors and stress is represented by the length of the radial lines on the weight bearing area. The dot on the weight bearing area corresponds to the position of the stress pole. As the stress pole lies within the weight bearing area, the stress pole is also the point where stress attains its maximal value

There have been singular cases of measurement of hip stress distribution with instrumented prosthesis [9]; valuable information, particularly regarding different everyday activities, was obtained. It was found that stress was non-zero in bedrest, while it was several folds increased in some common activities such as standing up from a low chair [9]. Studies on instrumented prosthesis improved understanding the role of the hip mechanics, however, these invasive and time consuming methods are not suitable for determination of hip stress in clinical practice. For the use in clinical practice and in studies of larger populations of hips, simple methods that can determine biomechanical parameters in a reasonable time are needed. For this purpose, a simple method based on the mathematical models has been developed to assess stress from the
X-ray images; the model HIPSTRESS for calculation of resultant hip force in a one legged stance was developed [12], [13] based on equations of mechanical equilibrium. Standing on one leg was considered the representative body position of everyday life activities [4].

Using the HIPSTRESS model, the arguments in favour of the hypothesis of increased hip stress in dysplastic hips were provided by a study where the peak hip stress on the load bearing area was determined in a population of hips with diagnosis of dysplasia and compared to hips with no record of disease; the two-fold difference in the average peak stress $p_{\max }$ normalized with respect to body weight $W_{\mathrm{B}}, p_{\max } / W_{\mathrm{B}}$ $\left(7100 / \mathrm{m}^{2}\right.$ in dysplastic hips vs. $3500 / \mathrm{m}^{2}$ in normal hips) was statistically highly significant $(p<0.001)$ [18].

The HIPSTRESS model is based on a 3-dimensional configuration of a representative adult hip and pelvis measured in a cadaver [12], [5] which are rescaled according to the geometry of the hip of interest. In the model [12], [13], the body is composed of two segments: the loaded leg and the rest of the body. The input are the positions of the attachment points of muscles that connect both segments and are active in this particular body position (i.e., gluteus minimus, gluteus medius, piriformis, tensor fasciae latae and rectus femoris). The model parameters represent the shape of the pelvis and the proximal femur (Fig. 1A: pelvis height $H$, pelvis width $C$, interhip distance $l$, position of the point on the greater trochanter with respect to the centre of the hip articular sphere in the coordinate system of the femur $\left(x_{\mathrm{T}}\right.$ and $\left.z_{\mathrm{T}}\right)$ ) and body weight $\mathbf{W}_{\mathrm{B}}$. Muscles are described as acting in straight lines connecting the origo and insertio while the magnitudes of muscle forces are proportional to their cross sectional areas and the exerted tensions [12], [13]. As there are more unknowns than equations available for mechanical equilibrium, additional assumptions are applied to reduce the number of unknowns [13]. It is, therefore, assumed that the muscles located close together develop the same muscle tension, thereby reducing the number of unknown muscle tensions to 3 . The results of the mechanical equilibrium are 3 tensions and 3 components of the resultant hip force $\mathbf{R}$ that acts in the origin of the coordinate system (the centre of the articular sphere).

The model HIPSTRESS for the contact hip stress [14] uses the resultant hip force $\mathbf{R}$, the radius of the joint sphere $r$ and the centre-edge angle $\vartheta_{\mathrm{CE}}$ as input parameters. The distribution of stress is obtained by requirement that the resultant hip force is an integral of stress $p$ 


$$
\mathbf{R}=\int p \mathbf{d A}
$$

where $\mathbf{d A}$ is the area element and integration is performed over the load bearing surface. Area element vector has the direction normal to the area element. While the HIPSTRESS model for resultant hip force is limited to one legged stance, the model HIPSTRESS for contact hip stress can be used to determine stress also in other body positions and activities. In principle, $\mathbf{R}$ can be obtained by measurements or by other models. Besides the peak value of stress on the loaded area $p_{\max }$, the relevant biomechanical parameters are: index of the hip stress gradient $G_{p}$, functional angle of the weight bearing area $\vartheta_{f}$, size of the weight bearing area $A$ and the position of the stress pole $\Theta$.

The models HIPSTRESS for force and for stress were both used to analyse dysplastic hips [18], [20], hips after the Perthes disease [15], [19] and hips that underwent operations [23]. The HIPSTRESS models proved useful in explaining the early cartilage degeneration in pathologies connected to either elevated peak stress and/or positive sign of the hip stress gradient index.

In clinical practice, it is useful to have a well-defined parameter that distinguishes health from disease. In any parameter there is however a range of values that are indecisive due to measuring errors and limitations of the model. Well-defined parameters have smallest possible range of indecisive cases. In this work we tried to outline a biomechanical parameter which could be predictive for increased probability of hip cartilage degeneration due to elevated contact hip stress. Previous works considered either the peak stress on the load bearing area $p_{\max }$ (in $\mathrm{MPa}$ ), or peak stress normalized with respect to body weight $p_{\max } / W_{\mathrm{B}}$ (this parameter gives the impact of the hip and pelvis geometry on stress) or hip stress gradient index (in $\mathrm{MPa} / \mathrm{m}$ ). However, as 2-dimensional images, e.g., X-ray, are often used to assess the hip and pelvis geometry, the scaling procedure applied in the HIPSTRESS model for force [10] neglects the anteroposterior dimension of the body. This may cause errors. Furthermore, the previously used parameters $p_{\max }$ and $p_{\max } / W_{\mathrm{B}}$ are inversely proportional to the square of the radius of the joint sphere which can not always be determined due to unknown magnification of the image. It is the purpose of this work to improve the HIPSTRESS method by suggesting a more convenient model for the force that would be independent of the size of the image and find the stress parameters with well-defined range for prediction of early coxarthritis risk.

\section{Methods}

\subsection{The one-muscle hipstress model for resultant hip force}

In the model, the body is composed of two segments: (the loaded leg (lower segment) and the rest of the body (upper segment)) which are connected by the joint. To maintain the equilibrium by standing on one leg, the centre of the mass of the other parts of the body should be moved towards the loaded leg which is obtained by slight rotation of the pelvis. For simplicity, we neglected this and assumed the pelvis as levelled. We considered that all the forces in the hip lie in the frontal plane of the body through the centres of both femoral heads. We considered three forces acting on the upper segment: the weight of the upper segment $\left(\mathbf{W}_{\mathrm{B}}-\mathbf{W}_{\mathrm{L}}\right)$, where $\mathbf{W}_{\mathrm{B}}$ is the weight or the entire body and $\mathbf{W}_{\mathrm{L}}$ is the weight of the loaded leg, the effective force of the muscles pulling the upper segment down towards the loaded leg $\mathbf{F}$, and the resultant hip force $\mathbf{R}$ which equilibrates the weight of the upper segment and the muscle force,

$$
\mathbf{R}=\left(\mathbf{W}_{\mathrm{B}}-\mathbf{W}_{\mathrm{L}}\right)+\mathbf{F} \text {. }
$$

The origin of the coordinate system is taken at the centre of the articular sphere fitting the contour of the femoral head under the acetabular roof, the $x$ axis points in the medial direction, the $z$ axis points to the vertical direction and the $y$ axis points out of the anteroposterior plane. The forces are represented by their inclination angles with respect to the vertical direction, $\mathbf{W}_{\mathrm{B}}-\mathbf{W}_{\mathrm{L}}=\left(0,0,-\left(W_{\mathrm{B}}-W_{\mathrm{L}}\right)\right), \mathbf{F}=\left(-F \sin \vartheta_{F}, 0\right.$, $\left.-F \cos \vartheta_{F}\right), \mathbf{R}=\left(R \sin \vartheta_{R}, 0, R \cos \vartheta_{R}\right)$ and the respective moment arms are $\mathbf{r}_{W_{\mathrm{B}}-W_{\mathrm{L}}}=\left(l / 2,0, z_{\mathrm{CM}}\right), \mathbf{r}_{F}=$ $\left(-x_{F}, 0, z_{F}\right)$ and $\mathbf{r}_{R}=(0,0,0)$ (Fig. 1A). As the problem is mapped to two dimensions, the $x$ and $z$ components for the equilibrium of forces (Eq. (2)) yield the system

$$
R \sin \vartheta_{R}=F \sin \vartheta_{F}
$$

and

$$
R \cos \vartheta_{R}=\left(W_{\mathrm{B}}-W_{\mathrm{L}}\right)+F \cos \vartheta_{F}
$$

The equilibrium of torques

$$
\mathbf{M}_{W_{\mathrm{B}}-W_{\mathrm{L}}}+\mathbf{M}_{F}+\mathbf{M}_{R}=0
$$

where $\mathbf{M}_{i}=\mathbf{r}_{i} \times \mathbf{F}_{i}$ and index $i$ runs over all muscles included, gives 2 trivial equations for the $x$ and $z$ components while for the $y$ component the equilibrium yields 


$$
F\left(x_{F} \cos \vartheta_{F}+z_{F} \sin \vartheta_{F}\right)=l\left(W_{\mathrm{B}}-W_{\mathrm{L}}\right) .
$$

The geometrical parameters $\vartheta_{F}, x_{F}, z_{F}$ and $l / 2$ can be determined from images (Fig. 1A) as described below. The weight of the supporting leg is approximated by $1 / 7$ of the body weight [12], [13],

$$
W_{L}=W_{\mathrm{B}} / 7 \text {. }
$$

The above arguments yield a set of 3 equations with 3 unknowns $\left(F, \vartheta_{R}\right.$ and $\left.R\right)$ with a straightforward solution

$$
\begin{aligned}
& F / W_{\mathrm{B}}=3 l / 7\left(x_{F} \cos \vartheta_{F}+z \sin \vartheta_{F}\right), \\
& \tan \vartheta_{R}=\sin \vartheta_{F} /\left(\cos \vartheta_{F}+6 W_{\mathrm{B}} / 7 F\right)
\end{aligned}
$$

and

$$
R / W_{\mathrm{B}}=F / W_{\mathrm{B}} \sin \vartheta_{F} / \sin \vartheta_{R}
$$

The components of forces are normalized by body weight which is of practical importance because the body weight is sometimes not known. The forces are proportional to the body weight, therefore, the normalized parameters express the contribution of the geometry to the forces and are of interest per se. The force Equations (8)-(10) were derived from the mechanical equilibrium of the upper body segment, however, their reaction forces are acting also to the lower body segment (the loaded leg) and are, therefore, connected with the force that the body imposes to the ground which can be measured [25]. In the following research we are planning to take the reaction forces into consideration, as we are focusing on the distribution of stress over the femoral head.

\subsection{Nomogram for stress within the HIPSTRESS model}

To determine stress distribution over the load bearing area of the femoral head with the HIPSTRESS model, Eq. (1) must be solved by considering the dependence of components of $R$ obtained by solving Eqs. (8)-(10).

In the model, the femoral head is represented by a spherical surface with the radius that fits the head at the region of load by the acetabulum. The acetabulum (which appears as a hemispherical shell) is represented in the model by a spherical surface with the radius larger than the radius of the femoral head by the thickness of unloaded cartilage. It is assumed that, in the reference state (the unloaded hip), the centres of the two spherical surfaces coincide. With the load, they are mutually displaced and the cartilage between them is squeezed. The articular sphere is defined as an abstract spherical surface representing the femoral head and the acetabulum, with the origin at the centre of the unloaded hip and with the radius of the femoral head (Fig. 2). The point at the articular sphere corresponding to the direction of the closest approach of the femoral head and the acetabulum is called the stress pole (Fig. 2). The Hook's law is applied by assuming that the stress is proportional to strain whereas geometrical consideration yields the cosine dependence on the space angle $\gamma[1]$

$$
p=p_{0} \cos \gamma,
$$

where $p_{0}$ is stress at the stress pole. The cosine dependence however, ceases when the stress reaches the value zero, since negative values of stress are then unphysical. The points where the stress reaches the value zero were, therefore, taken as the medial border of the load bearing area. However, the pole is not always located within the load bearing area as the load bearing area is limited also by the structures of the bones. Within the HIPSTRESS model, the lateral border of the load bearing area is modelled by intersecting the articular sphere surface by a plane through the centre of the sphere which is inclined with respect to the vertical by the centre-edge angle $\vartheta_{\mathrm{CE}}$ (Fig. 2). Within such configuration, in the one legged stance, the pole is expected to lie close to the lateral border of the load bearing area while the medial border is reached when the cosine function attains the value 0 , i.e., for $\pi / 2$ from the pole (Fig. 2). Due to a spherical shape of the joint, a coordinate system can always be found, in which the shape of the load bearing area is symmetrical. This coordinate system is defined by the direction of the resultant hip force $\mathbf{R}$.

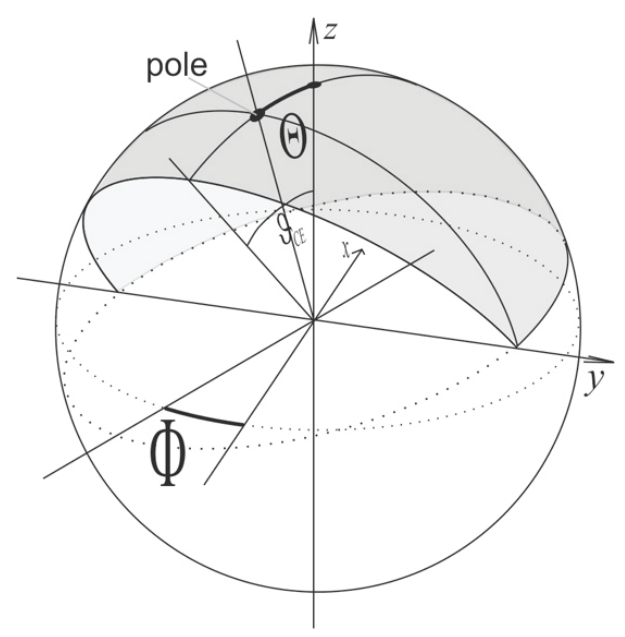

Fig. 2. A schematic of the weight bearing area (shaded) as a part of the articular sphere. The position of the stress pole is given by the angles $\Theta$ and $\Phi$ as indicated 
The unknowns of the three components of Eq. (1) are the two angles determining the position of the pole on the articular sphere (denoted by $\Phi$ and $\Theta$, respectively) (Fig. 2), and the constant $p_{0}$. In the case of HIPSTRESS model, the resultant hip force lies in the frontal plane of the body [18] whereas the symmetry plane is the sagittal plane and the stress pole lies in the frontal plane consistent with $\Phi=0$ or $\pi$ [14]. We chose to represent the solution for $\Phi$ as the sign in front of the inclination of the vector pointing from the origin of the coordinate system to the stress pole towards the vertical axis $z$ (the angle $\Theta$ ). Thus, the position of the stress pole is given as $\Theta$ positive in the lateral direction and negative in the medial direction. The values of $\Theta$ and $p_{0}$ are obtained by the solution of the system of equations deriving from Eqs. (1) and (11), [14]

$$
\begin{aligned}
\tan \left(\vartheta_{R}+\Theta\right) & =\cos ^{2}\left(\vartheta_{\mathrm{CE}}-\Theta\right) /\left(\pi / 2+\left(\vartheta_{\mathrm{CE}}-\Theta\right)\right. \\
& \left.+\sin \left(2\left(\vartheta_{\mathrm{CE}}-\Theta\right)\right) / 2\right)
\end{aligned}
$$

and

$$
p_{0} / W_{\mathrm{B}}=3 R \sin \left(\vartheta_{R}+\Theta\right) /\left(2 W_{\mathrm{B}} r^{2} \cos ^{2}\left(\vartheta_{\mathrm{CE}}-\Theta\right)\right) .
$$

We introduced new variables

$$
\vartheta_{\mathrm{RCE}}=\left(\vartheta_{R}+\vartheta_{\mathrm{CE}}\right) / 2
$$

and

$$
\vartheta_{\mathrm{H}}=\Theta-\left(\vartheta_{\mathrm{CE}}-\vartheta_{R}\right) / 2
$$

into Eq. (12) to yield

$$
\begin{gathered}
\tan \left(\vartheta_{\mathrm{RCE}}+\vartheta_{\mathrm{H}}\right) \\
=\cos ^{2}\left(\vartheta_{\mathrm{RCE}}-\vartheta_{\mathrm{H}}\right) /\left(\pi / 2+\left(\vartheta_{\mathrm{RCE}}-\vartheta_{\mathrm{H}}\right)\right. \\
\left.+\sin \left(2\left(\vartheta_{\mathrm{RCE}}-\vartheta_{\mathrm{H}}\right)\right) / 2\right) .
\end{gathered}
$$

There are two parameters in Eq. (16): angles $\vartheta_{\mathrm{RCE}}$ and $\vartheta_{\mathrm{H}}$. The angle $\vartheta_{\mathrm{RCE}}(\mathrm{Eq}$. (14)) is a result of the input data obtained from the model for force $\left(\vartheta_{R}\right)$ and the measurement of the angle $\vartheta_{\mathrm{CE}}$ from the image. Using these data, Eq. (16) was solved to obtain $\vartheta_{\mathrm{H}}$. It can be seen that Eq. (16) is a nonlinear equation and must be solved numerically. The value of $\vartheta_{\mathrm{H}}$ can be conveniently assessed from the nomogram (Fig. 3) which documents the solutions of Eq. (16) for different values of $\vartheta_{\text {RCE. }}$ For construction of the nomogram, Eq. (16) was solved by using the Newton method implemented in a Turbo Pascal, Borland International, version 7.0.

After obtaining $\vartheta_{\mathrm{H}}$ by solving Eq. (16), the position of the stress pole was obtained by rearranging Eq. (15),

$$
\Theta=\vartheta_{\mathrm{H}}+\left(\vartheta_{\mathrm{CE}}-\vartheta_{R}\right) / 2
$$

and the value of stress at the pole $p_{0}$ was calculated by introducing the new variables (14) and (15) into Eq. (13), $p_{0} r^{2} / W_{\mathrm{B}}=3 R \sin \left(\vartheta_{\mathrm{RCE}}+\vartheta_{\mathrm{H}}\right) /\left(2 W_{\mathrm{B}} \cos ^{2}\left(\vartheta_{\mathrm{RCE}}-\vartheta_{\mathrm{H}}\right)\right)$.

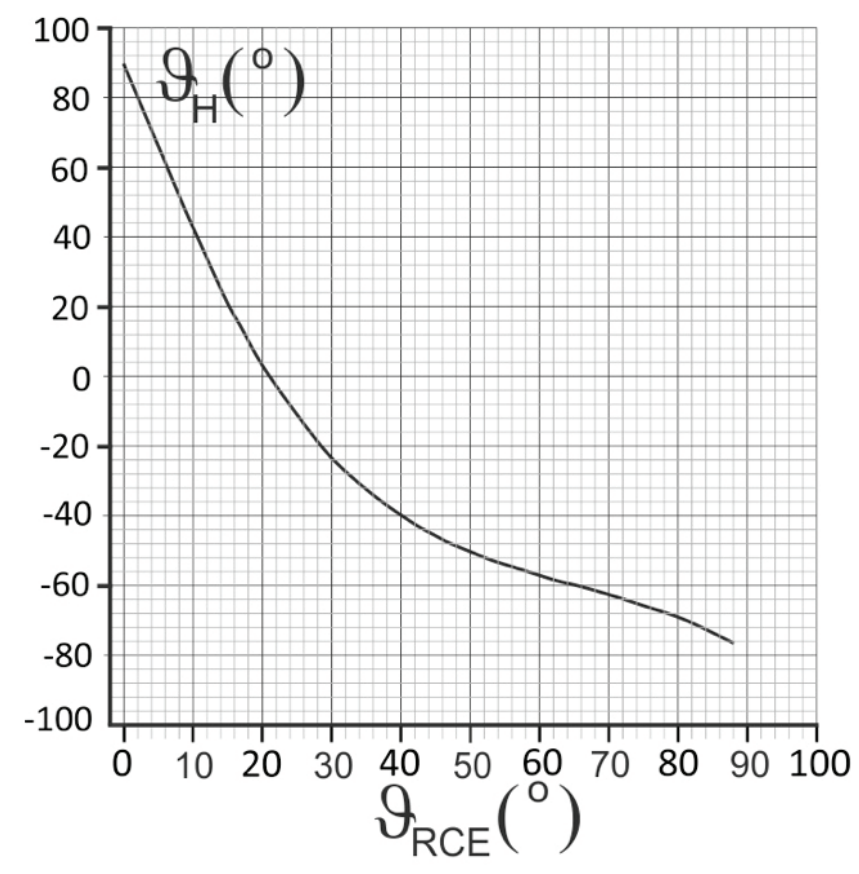

Fig. 3. A nomogram for solving Eq. (16) to determine the position of the stress pole $\Theta$ in the HIPSTRESS model for stress

Dimensionless biomechanical parameters that characterize the hip within the one-muscle HIPSTRESS model are the magnitude of the muscle force normalized by body weight $F / W_{\mathrm{B}}$, the magnitude of the resultant hip force normalized by body weight $R / W_{\mathrm{B}}$, the inclination of the resultant hip force $\vartheta_{R}$, the peak contact hip stress normalized by body weight and radius of the articular sphere $p_{\max } r^{2} / W_{\mathrm{B}}$,

$$
\begin{gathered}
p_{\max } r^{2} / W_{\mathrm{B}}=p_{0} r^{2} / W_{\mathrm{B}} \text { for }\left(\Theta \leq \vartheta_{\mathrm{CE}}\right), \\
p_{\max } r^{2} / W_{\mathrm{B}}=p_{0} r^{2} \cos \left(\vartheta_{\mathrm{CE}}-\Theta\right) \text { for }\left(\Theta>\vartheta_{\mathrm{CE}}\right),
\end{gathered}
$$

the position of the stress pole $\Theta$, the hip stress gradient index normalized by body weight and radius of the articular sphere $G_{p} r^{3} / W_{\mathrm{B}}$,

$$
G_{p} r^{3} / W_{\mathrm{B}}=-p_{0} r^{2} \sin \left(\vartheta_{\mathrm{CE}}-\Theta\right),
$$

and the functional angle of weight bearing $\vartheta_{f}$

$$
\vartheta_{f}=\pi / 2+\vartheta_{\mathrm{CE}}-\Theta .
$$

When analysing dysplastic and normal hips by the HIPSTRESS models, it was suggested that the classification parameter is the sign of the hip stress gradient index $G_{p}$, which depends on the difference $\left(\vartheta_{\mathrm{CE}}-\Theta\right)$ (Eq. (20)) [21]. Hips with negative $G_{p}$ are normal and hips with positive $G_{p}$ are considered dysplastic. It was found that this classification correlated better with the 
clinical evaluation (Harris hip score) than the standard classification according to the centre edge angle $\vartheta_{\mathrm{CE}}$ [21]. According to the HIPSTRESS classification, dysplastic hips are those hips in which the stress pole lies outside the weight bearing area $\left(\Theta>\vartheta_{\mathrm{CE}}\right)$.

\subsection{Determination of the geometrical and biomechanical parameters by using a radiograph of pelvis and proximal femora}

The geometrical parameters needed by the models are shown in Fig. 1A. As the parameters of the one-muscle model are invariant to scale, the unit size is not required. The picture should be leveled, and the circles should be fitted to that region of both femoral heads where the highest load is expected. These circles represent articular spheres of the two hips. A horizontal line should be drawn to assess the interhip distance (the distance between the two head centres). To describe the hip, a coordinate system was introduced with the origin at the centre of the femoral head. The positive $x$ axis points medially and the positive $z$ axis points upwards. To estimate the attachment point of the effective muscle on the pelvis, we used half of the horizontal distance from the femora $\left(x_{F}=C / 2\right)$ and three quarters of the pelvic height $\left(z_{F}=3 H / 4\right)$, where $C$ and $H$ are the parameters of the HIPSTRESS model for force [3]. The point on the trochanter was obtained in the coordinate system of the femur. The $z$-axis of the femur is directed along the bone canal while the $x$ axis is perpendicular to it. The highest point on the greater trochanter and the most lateral point on the pelvis are connected by a straight line and a symmetral is drawn. The attachment point of the effective muscle was estimated at the point where this symmetral crosses the contour of the greater trochanter. The inclination of the line connecting both imaginary attachment points with respect to the vertical defines the angle $\vartheta_{F}$. The centre-edge angle was obtained by drawing a line that connects the centre of the articular sphere with the most lateral point of the bony contour. The inclination of this line with respect to the vertical defines the centre-edge angle $\vartheta_{\mathrm{CE}}$.

Measurement of geometrical parameters for the right hip is shown in Fig. 1B and the calculated biomechanical parameters are represented in Fig. 1C. Solution of Eqs. (8)-(10) by using the data from Fig. 3B $(l / 2=$ $70.4, x_{F}=C / 2=18.2, z_{F}=3 H / 4=80.9, \vartheta_{F}=15.11$ ) gives $F / W_{\mathrm{B}}=1.56, \vartheta_{R}=9.8^{\circ}$ and $R / W_{\mathrm{B}}=2.40$. The corresponding values obtained by the full HIPSTRESS model for resultant hip force with parameters $(l / 2$, $C, H$ and $x_{\mathrm{T}}$ ) were assessed from nomograms [3] $\left(\vartheta_{R}=10^{\circ}\right.$ and $\left.R / W_{\mathrm{B}}=2.60\right)$. The difference in $\vartheta_{R}$ was very small, so with $\vartheta_{\mathrm{CE}}=23.62^{\circ}$, the parameter $\vartheta_{\mathrm{RCE}}$ (Eq. (11)) was almost the same in both models $\left(17^{\circ}\right)$ while the corresponding $\vartheta_{\mathrm{H}}$ assessed from Fig. 3 was $14^{\circ}$. Position of the stress pole $\Theta$ calculated by using Eq. (17) was $21^{\circ}$. As the pole lies within the load bearing area $\left(\Theta\right.$ is smaller than $\left.\vartheta_{\mathrm{CE}}\right)$, the value of stress at the pole calculated by Eq. (18) was also the value of peak stress. The dimensionless peak stress (Eq. (18)) $p_{\max } r^{2} / W_{\mathrm{B}}$ is 1.85 in the one-muscle model and 2.00 in the full HIPSTRESS model, the dimensionless gradient index (Eq. (20)) $G_{p} r^{3} / W_{\mathrm{B}}$ is -0.77 in the one-muscle model and -0.62 in the full HIPSTRESS model. The functional angle of the weight bearing area (Eq. (21)) $\vartheta_{f}$ is $93^{\circ}$ in both models. It can be seen that the one muscle model in the presented hip differed within $10 \%$ from the full HIPSTRESS model. The comparison was made for a hip contralateral to the hip that was subjected to Perthes disease in childhood. This hip was considered normal. Good agreement between the two models should however be confirmed also in studies involving larger numbers of hips and different pathologies.

\section{Results and discussion}

\subsection{Dimensionless peak stress as a parameter for assessment of risk for early hip arthritis}

To validate the biomechanical parameters of the HIPSTRESS model, we analysed X-ray images of 172 hips that were in the childhood subjected to Perthes disease [15], [19]. Although the etiology of this disease is not completely understood, it is acknowledged that in the acute phase, defective function of the vascular system causes inadequate blood supply to the femoral head [15]. It was found that this population developed a relatively large number of dysplastic hips in adulthood, compared to a normal population [19]. The dimensions of the hips in these studies were known as the patients were imaged with a hip endoprosthesis head of a known radius between their legs. This enabled also calculation of non-normalized parameters. Resultant hip 
force was calculated by the full HIPSTRESS model. In the present study, the hips were divided into two groups: dysplastic hips and normal hips. Two classification criteria for dysplasia were applied to the same population: the hip stress gradient index [20] $\left(G_{p}>0\right) ; 74$ hips were dysplastic and 98 hips were normal) and centre-edge angle [7] $\left(\vartheta_{\mathrm{CE}}<20^{\circ}\right) ; 53$ hips were dysplastic and 119 hips were normal). Two parameters were compared: new dimensionless peak stress $p_{\max } r^{2} / W_{\mathrm{B}}$ and peak stress normalized by body weight $p_{\max } / W_{\mathrm{B}}$ which was previously used to evaluate cohorts with different hip pathologies [15], [18]-[20], [22], [23].

It can be seen in Table 1 that according to the HIPSTRESS classification of dysplasia $\left(G_{p}>0\right)$, all biomechanical parameters statistically significantly differed in the two populations with all biomechanical parameters being more favourable in normal hips, while, according to centre-edge angle classification $\left(\vartheta_{\mathrm{CE}}<20^{\circ}\right)$, the difference in the direction of the resultant hip force was not revealed while in other parameters the results were similar to the results of the HIPSTRESS classification. The body weight did not show statistically significant difference between the two populations and the femoral head radius was larger (more favourable) in dysplastic hips than in normal hips due to compensatory effects during the development of the hip after the Perthes disease, according to both classifications [15]. It seems that both classifications and all biomechanical parameters are able to explain the difference between hips at increased risk for early coxarthritis development and normal hips, on the population level. However, to be able to assess the risk in an individual, the relevant parameter should clearly distinguish health from disease.

In Figure 4, the peak stress parameters $p_{\max } r^{2} / W_{\mathrm{B}}$ and $p_{\max } / W_{\mathrm{B}}$ distributed with respect to the radius of the femoral head are shown. The crosses pertain to dysplastic hips and the dots pertain to normal hips. The grey zone is defined as the area of the diagram where both types of hips coexist, i.e., where the model cannot distinguish between the two groups. The grey zone contained 6\% of all hips in Panel A, $19 \%$ in Panel B, $81 \%$ in Panel C and $89 \%$ in Panel D. (The dimensionless parameter $p_{\max } r^{2} / W_{\mathrm{B}}$ (panels $A$ and $B$ ) exhibited much smaller grey zone of indecisive cases than the peak stress normalized by body weight (panels $\mathrm{C}$ and $\mathrm{D}$ ). The classification with respect to hip stress gradient index $\left(G_{p}>0\right)$ (panels $\mathrm{A}$ and $\mathrm{C}$ ) yielded smaller grey zone than classification according to centre edge angle $\left(\vartheta_{\mathrm{CE}}<20^{\circ}\right)$ (panels B and D). It can be seen (panels A, B) that dimensionless peak stress $p_{\max } r^{2} / W_{\mathrm{B}}$ presented a constant value limit that applied to hips of a wide range of sizes. However, this limit could be better envisaged by using the HIPSTRESS classification $\left(G_{p}>0\right)$ than the centre-edge angle classification $\left(\vartheta_{\mathrm{CE}}<20^{\circ}\right)$. The results shown in Fig. 4 indicate a well-defined threshold for an increased risk of early coxarthritis development by the HIPSTRESS parameter

$$
H=p_{\max } r^{2} / W_{\mathrm{B}}(\text { border })=2 .
$$

Table 1. Biomechanical parameters of hips with increased risk for early coxarthritis represented by dysplastic hips and normal hips according to two classifications (by centre-edge angle $\vartheta_{\mathrm{CE}}$ and by hip stress gradient index $G_{p}$ ). Asterisks denote the biomechanically more favorable values

\begin{tabular}{|c|c|c|c|c|c|c|c|c|c|c|}
\hline $\begin{array}{l}\text { Classification } \\
\text { criterion }\end{array}$ & \multicolumn{5}{|c|}{$\vartheta_{\mathrm{CE}}<20^{\circ}$} & \multicolumn{5}{|c|}{$G_{p}>0$} \\
\hline Population & $\begin{array}{l}\text { Hips wit } \\
\text { risk for } \\
(N\end{array}$ & $\begin{array}{l}\text { rreased } \\
\text { rthritis } \\
\text { ) }\end{array}$ & $\begin{array}{l}\text { Nor } \\
(N=\end{array}$ & & $\begin{array}{c}\text { Statistical } \\
\text { significance } \\
\text { of the difference }\end{array}$ & $\begin{array}{l}\text { Hips wit } \\
\text { risk for } \\
\qquad(N\end{array}$ & $\begin{array}{l}\text { creased } \\
\text { arthritis } \\
\text { 4) }\end{array}$ & & & $\begin{array}{c}\text { Statistical } \\
\text { significance } \\
\text { of the difference }\end{array}$ \\
\hline Parameter & Mean & SD & Mean & $\mathrm{SD}$ & $p(t$-test $)$ & Mean & SD & Mean & $\mathrm{SD}$ & $p(t$-test $)$ \\
\hline$W_{\mathrm{B}}[\mathrm{N}]$ & 802 & 194 & 832 & 189 & 0.35 & 811 & 177 & 831 & 201 & 0.49 \\
\hline$\vartheta_{\mathrm{CE}}\left[{ }^{\circ}\right]$ & 12.12 & 5.20 & 29.91* & 5.82 & $10^{-43}$ & 15.19 & 6.67 & $31.39 *$ & 5.27 & $10^{-40}$ \\
\hline$r[\mathrm{~cm}]$ & $3.23 *$ & 0.53 & 2.65 & 0.34 & $10^{-14}$ & $3.11 *$ & 0.52 & 2.62 & 0.33 & $10^{-11}$ \\
\hline$\vartheta_{R}\left[{ }^{\circ}\right]$ & 8.98 & 1.94 & 8.85 & 2.00 & 0.68 & 8.52 & 1.97 & $9.17 *$ & 1.95 & 0.03 \\
\hline$\Theta\left[^{\circ}\right]$ & 41.29 & 9.14 & $16.37 *$ & 7.85 & $10^{-41}$ & 37.58 & 9.87 & $13.82 *$ & 6.00 & $10^{-44}$ \\
\hline$R / W_{\mathrm{B}}$ & 2.76 & 0.34 & $2.55^{*}$ & 0.21 & $10^{-6}$ & 2.75 & 0.30 & $2.51 *$ & 0.19 & $10^{-9}$ \\
\hline$p_{\max } / W_{\mathrm{B}}\left[\mathrm{m}^{-2}\right]$ & 3889 & 1491 & $2591 *$ & 673 & $10^{-12}$ & 3673 & 1371 & $2476^{*}$ & 587 & $10^{-12}$ \\
\hline$p_{\max }[\mathrm{MPa}]$ & 2.97 & 1.12 & $2.23 *$ & 1.06 & $10^{-04}$ & 2.86 & 1.05 & $2.15^{*}$ & 1.10 & $10^{-04}$ \\
\hline$p_{\max } r^{2} / W_{\mathrm{B}}$ & 3.98 & 1.66 & $1.78^{*}$ & 0.38 & $10^{-28}$ & 3.53 & 1.58 & $1.64 *$ & 0.24 & $10^{-22}$ \\
\hline$G_{p} r^{3} / W_{\mathrm{B}}$ & 3.05 & 3.47 & $-0.37 *$ & 0.39 & $10^{-19}$ & 2.25 & 3.20 & -0.50 & 0.29 & $10^{-14}$ \\
\hline
\end{tabular}


Normal hips have $H<2$ and dysplastic hips have $H>2$. The border was identified by a population of dysplastic hips, however, there are also other pathologies in which stress is elevated to a similar extent and the criterion for the risk of early coxarthritis $(H>2)$ is expected to apply also to these hips and other pathologies. The borderline value $H=2$ is a result of a consistent use of the HIPSTRESS models to assess the hips. It would be of interest to see whether it could be predictive also by using other models and independent distribution parameters as well as different cohorts at risk for hip osteoarthritis.

It can be seen in Figs. 4A and B that hips with very large heads have on the average higher $p_{\max } r^{2} / W_{\mathrm{B}}$ whereas in parameter $p_{\max } / W_{\mathrm{B}}$ (Fig. $4 \mathrm{C}$ and $\mathrm{D}$ ), the increase in radius is not revealed. It can be seen that large radii that are developed after the Perthes disease keep the parameter $p_{\max } / W_{\mathrm{B}}$ low. As large heads in hips after the Perthes disease are connected to small centre-edge angles [15], this effect is considered a compensation mechanism in a developing hip, to keep stress as low as possible.
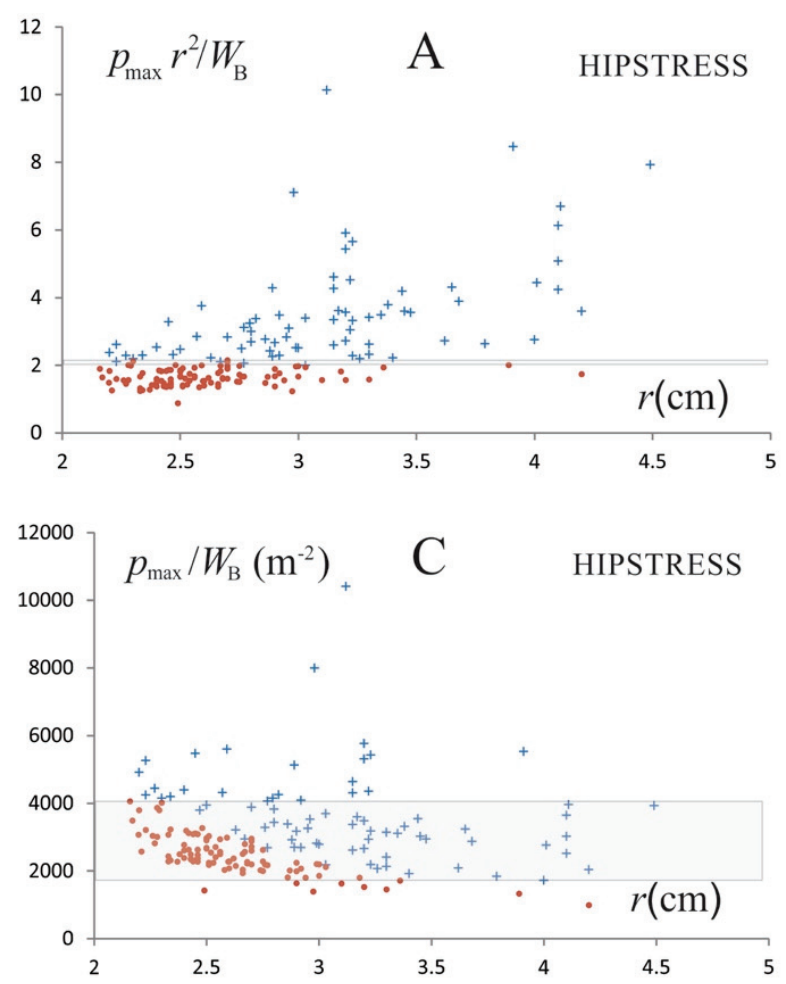

Distribution of the hips due to body weight is shown in Fig. 5. The dimensionless parameter $p_{\max } r^{2} / W_{\mathrm{B}}$ within the HIPSTRESS classification $\left(G_{p}>0\right)$ provides welldefined regions corresponding to hips with increased risk for coxarthritis development and normal hips (Fig. 5A) since the grey zone where both types are present is very small. On the other hand, when the same population is assessed with the previously used stress parameter $p_{\max } / W_{\mathrm{B}}$, in particular in combination with classification according to $\vartheta_{\mathrm{CE}}\left(\vartheta_{\mathrm{CE}}<20^{\circ}\right)$ (Figs. 5C, D), the grey zone where both types are present includes the majority of hips. While the parameter $p_{\max } / W_{\mathrm{B}}$ showed grouping of hips with respect to the radius of the articular surface $r$ (Fig. 4 C, D) (i.e., populations could be divided by some curve depending on $r$ ), the distribution over the body weight shown in Fig. 5B is random.

The mechanical hypothesis suggests that unrecognized unfavourable hip stress distribution is the cause of a considerable portion of primary arthritis cases. To reveal the causes, different approaches have been developed [2], [17], [11]. However, it might be that the risk is unrecognized because stress is not assessed in
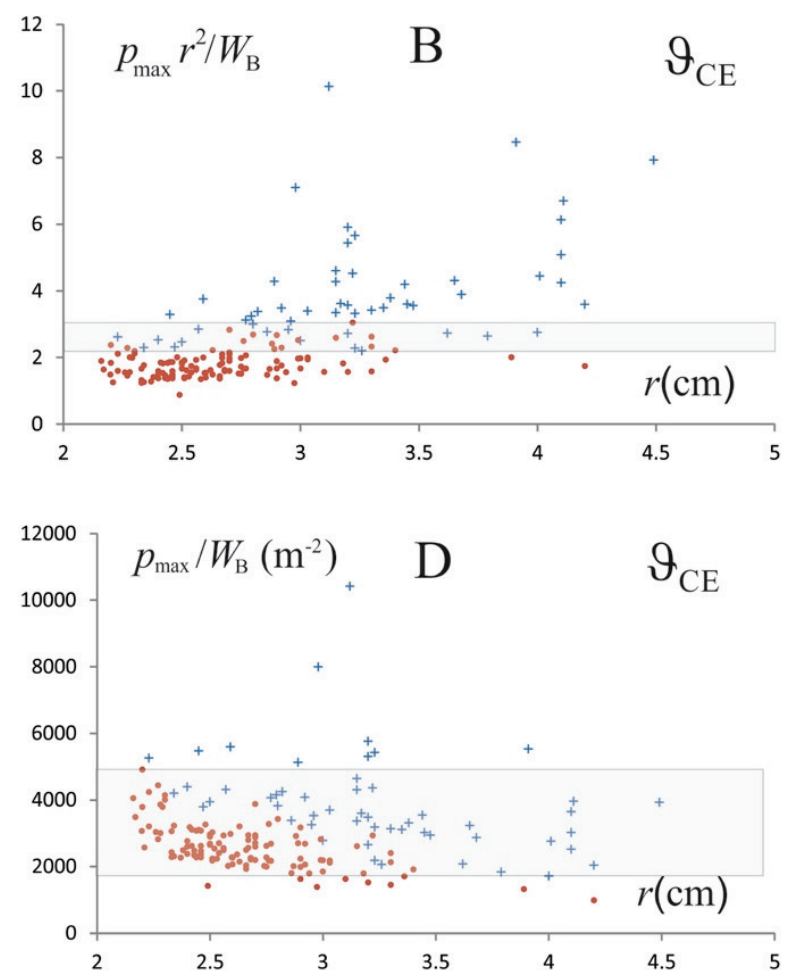

Fig. 4. Display of two different biomechanical parameters (dimensionless peak stress $p_{\max } r^{2} / W_{\mathrm{B}}$ (A and B) and peak stress normalized with respect to body weight $p_{\max } / W_{\mathrm{B}}\left(\mathrm{m}^{-2}\right)(\mathrm{C}$ and $\left.\mathrm{D})\right)$ for assessment of the risk for early coxarthritis development according to two criteria for dysplasia (HIPSTRESS $\left(G_{p}>0\right)$ (A and C) and $\left(\vartheta_{\mathrm{CE}}<20^{\circ}\right)(\mathrm{B}$ and D)). The biomechanical parameters of 172 hips, that were in the childhood subjected to Perthes disease and, therefore, included a relatively high portion of dysplastic hips into the population, are distributed with respect to the independent parameter: radius of the femoral head $r$. Crosses correspond to hips with increased risk for coxarthritis development (dysplastic hips) and dots correspond to normal hips. The grey area is defined as the zone where both types of hips coexist 

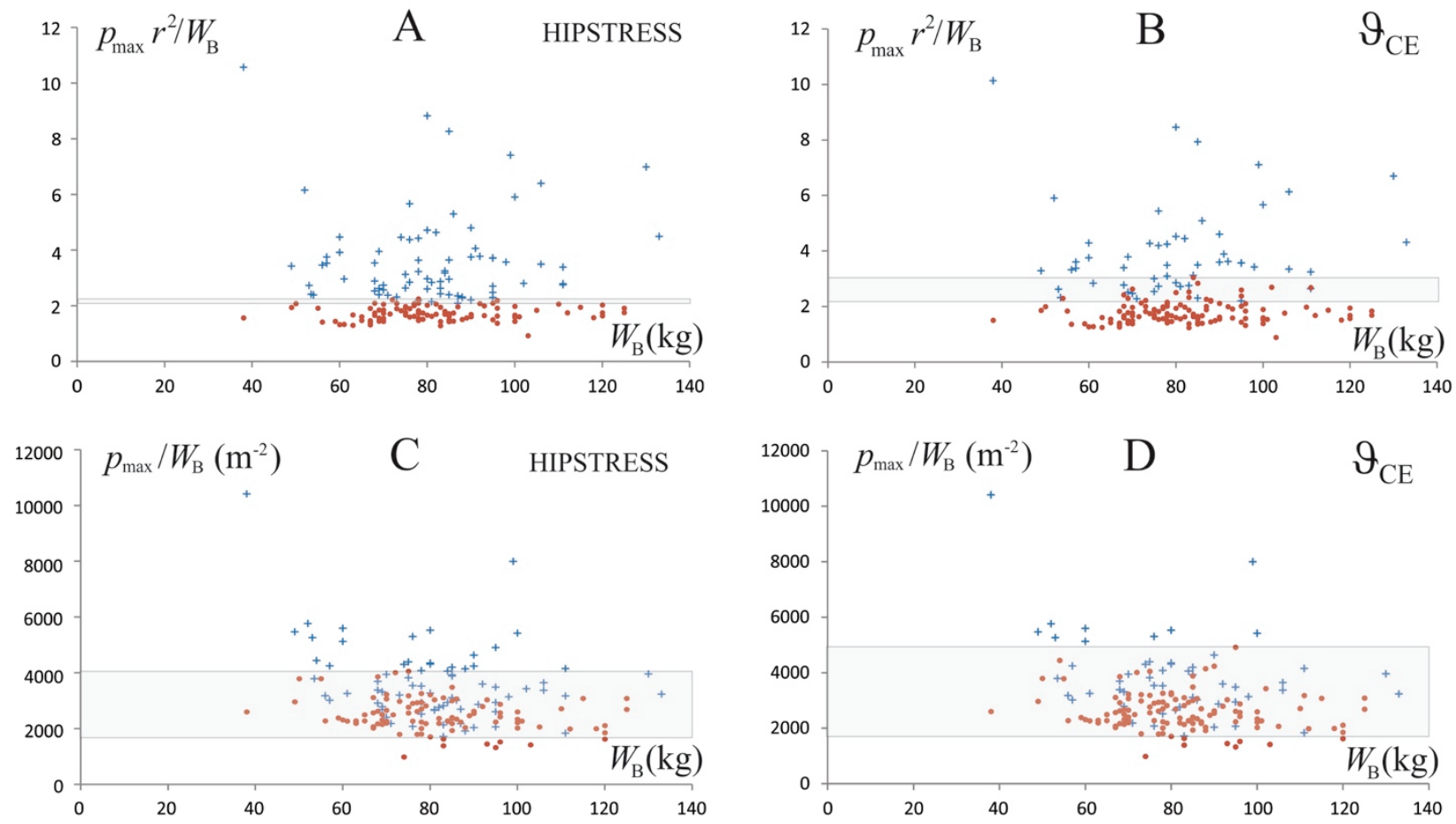

Fig. 5. Display of two different biomechanical parameters (dimensionless peak stress $p_{\max } r^{2} / W_{\mathrm{B}}(\mathrm{A}$ and B) and peak stress normalized with respect to body weight $p_{\max } / W_{\mathrm{B}}\left(\mathrm{m}^{-2}\right)(\mathrm{C}$ and $\mathrm{D})$ for assessment of the risk for early coxarthritis development according to two criteria for dysplasia (HIPSTRESS $\left(G_{p}>0\right)$ (A and C) and $\left(\vartheta_{\mathrm{CE}}<20^{\circ}\right)(\mathrm{B}$ and D)).

The biomechanical parameters of 172 hips that were in the childhood subjected to Perthes disease and, therefore, included in the population a relatively high portion of dysplastic hips, are distributed with respect to the body weight $W_{\mathrm{B}}$. Crosses correspond to hips with increased risk for coxarthritis development (dysplastic hips) and dots correspond to normal hips. The grey area is defined as the zone where both types of hips coexist

everyday clinical practice. Instead, geometrical parameters or morphological changes are used as its substitute [7]. These parameters document the situation but cannot include underlying mechanisms. The method presented in this work is based on the one legged stance as a representative body position. However, there are other positions and activities of importance in everyday life, in particular slow walking [14], [16].

\section{Conclusions}

Mathematical model is a tool which can be used to predict the coxarthritis risk. An applicable criterion for a given pathology should divide disease from health regardless of other properties. HIPSTRESS method has been previously developed and was further refined in this work with the purpose to be usable in personalized medicine. Based on analysis of hips subjected to Perthes disease in the childhood with a high incidence of hip dysplasia we outlined a criterion for an increased risk of coxarthritis development that could be used in clinical practice within the HIPSTRESS method: dimensionless peak stress on the load bearing area $(H)$. The borderline value over which the hips are at increased risk is $H=2$. In perspective, the parameter $H$ should be used in population studies. Also, userfriendly tools (software, applications) should be developed to enable simple and fast use of HIPSTRESS method in everyday clinical practice.

\section{Acknowledgements}

Authors acknowledge grants from Slovenian Research Agency P3-0388, J1-9162 and L3-2621.

\section{References}

[1] Brinckmann P., Frobin W., Hierholzer E., Stress on the articular surface of the hip joint in healthy adults and persons with idiopathic osteoarthrosis of the hip joint, J. Biomech., 1981, 14, 149-156, DOI: 10.1016/0021-9290(81)90021-x.

[2] Clarke S.G., Phillips A.T., Bull A.M., Evaluating a suitable level of model complexity for finite element analysis of the intact acetabulum, Comput. Methods Biomech. Biomed. Engin., 2013, 16, 717-724, DOI:10.1080/10255842.2011.633906. 
[3] Daniel M., Antolic V., Iglic A., Kralu-Iglic V., Determination of contact hip stress from nomograms based on mathematical model, Med. Eng. Phys., 2001, 23, 347-357, DOI:10.1016/ s1350-4533(01)00051-0.

[4] Debevec H., Pedersen D.R., Iglič A., Daniel M., One-legged stance as a representative static body position for calculation of hip contact stress distribution in clinical studies, J. Appl. Biomech., 2010, 26, 522-525, DOI:10.1123/jab.26.4.522.

[5] Dostal W.F., Andrews J.G., A three-dimensional biomechanical model of the hip musculature, J. Biomech., 1981, 14, 803-812, DOI: 10.1016/0021-9290(81)90036-1 13.

[6] Dragan S.L., Kuropka P., Kulej M., Gabryś P., Nikodem A., Changes in the mechanical properties of femoral cartilage tissue in advanced osteoarthritis, Acta Bioeng. Biomech., 2020, 22, 143-152, DOI: 10.37190/ABB-01463-2019-01.

[7] Gala J., Clohisy C., Beaulé P.E., Hip dysplasia in the young adult, J. Bone Joint Surg. Am., 2016, 98, 63-73, DOI: 10.2106/JBJS.O.00109.

[8] Ganz R., Leunig M., Leunig-Ganz K., Harris W.H., The etiology of osteoarthritis of the hip: an integrated mechanical concept, Clin. Orthop. Relat. Res., 2008, 466, 264-272, DOI: 10.1007/s11999-007-0060-z.

[9] Hodge W.A., Carlson K.L., Fijan R.S., Burgess R.G., RILEY P.O., HARRIS W.H. et al., Contact pressures from an instrumented hip endo-prosthesis, J. Bone Joint Surg. Am., 1989, 71, 1378-1386.

[10] Hornová J., Iglič A., Kralu-Iglič V., Pedersen D.R., DANIEL M., Effect of patient-specific model scaling on hip joint reaction force in one-legged stance - study of 356 hips, Acta Bioeng. Biomech., 2017, 19, 102-108, DOI: 15.5277/ABB00839-2017-02.

[11] HuA X., Li J., Wilcox R.K., Fisher J., JONES A.C., Geometric parameterisation of pelvic bone and cartilage in contact analysis of the natural hip: an initial study, Proc. Inst. Mech. Eng. H, 2015, 229, 570-580, DOI:10.1177/0954411915592656R.

[12] Iglič A., SRakar F., ANTOlič V., Kralu-IgliČ V., BatagelJ V., Mathematical analysis of Chiari osteotomy, Acta Orthop. Iugosl., 1990, 20, 35-39.

[13] IGLIČ A., SRAKAR F., AnTolič V., The influence of the pelvic shape on the biomechanical status of the hip, Clin. Biomech., (Bristol, Avon), 1993, 8, 223-224, DOI:10.1016/02680033(93)90019-E.

[14] IPAVec M., Brand R.A., Pedersen D.R., MavČIČ B., Kralu-IGLIČ V., IGLIČ A., Mathematical modelling of stress in the hip during gait, J. Biomech., 1999, 32, 1229-1235, DOI: 10.1016/s0021-9290(99)00119-0.

[15] Kocjančıč B., MoličniK A., Antolič V., MavČıČ B., KRALJ-IGLIČ V., VENGUST R., Unfavorable hip stress distri- bution after Legg-Calvé-Perthes syndrome: A 25-year follow-up of 135 hips, J. Orthop. Res., 2014, 32, 8-16, DOI: 10.1002/jor.22479.

[16] KolÁŘovÁ K., VodičKa T., BOZDĚCH M., REPKO M., 3D kinematic analysis of patients' gait before and after unilateral total hip replacement, Acta Bioeng. Biomech., 2020, 22, 165-171, DOI: 10.37190/ABB-01539-2020-04.

[17] Liu L., Siebenrock K., Nolte L.P., Zheng G.Y., Biomechanical Optimization-Based Planning of Periacetabular Osteotomy, V: Intelligent orthopaedics: artificial intelligence and smart image-guided technology for orthopaedics, G. Zheng, W. Tian, X. Zhuang (ur.), 2018, 1093, 157-168, DOI: 10.1007/978-981-13-1396-7_13.

[18] Mavčič B., Pompe B., Antolič V., Daniel M., Iglič A., KRALJ-IGLIČ V., Mathematical estimation of stress distribution in normal and dysplastic human hips, J. Orthop. Res., 2002, 20, 1025-1030, DOI: 10.1016/S0736-0266(02)00014-1.

[19] MoliČnik A., JanŠA J., KoCJanČIČ B., KRALJ-IGlič V., DOLINAR D., Secondary hip dysplasia increases risk for early coxarthritis after Legg-Calve-Perthes disease. A study of 255 hips, Comput. Methods Biomech. Biomed. Engin., 2019, 22, 1107-1115, DOI: 10.1080/10255842.2019.1634193.

[20] Pompe B., Daniel M., Sochor M., Vengust R., Kralu-IGLIČ V., IGLIČ A., Gradient of contact stress in normal and dysplastic human hips, Med. Eng. Phys., 2003, 25, 379-385, DOI: 10.1016/s1350-4533(03)00014-6.

[21] Pompe B., Antolič V., MAvČIČ B., Iglič A., Kralu-Iglič V., Hip joint contact stress as an additional parameter for determining hip dysplasia in adults: comparison with Severins classification, Med. Sci. Monit., 2007, 13, CR215-219.

[22] Tomaževič M., Kaiba T., KuREnt U., TrebšE R., Cimerman M., KRALJ-IGLIČ V., Hip stress distribution - predictor of dislocation in hip arthroplasties. A retrospective study of 149 arthroplasties, PLoS ONE, 2019, 14, e0225459, DOI: 10.1371/ journal.pone.0225459.

[23] Vukasinović Z., Spasovski D., KRALJ-Iglič V., MarinKovic-ERIC J., Seslija I., ŽIVKovič Z., Spasovski V., Impact of triple pelvic osteotomy on contact stress pressure distribution in the hip joint, Int. Orthop., 2013, 37, 95-98, DOI: 10.1007/ s00264-012-1727-y.

[24] Wiberg G., Studies on dysplastic acetabula and congenital subluxation of the hip joint: with special reference to the complication of osteoarthritis, Acta Chir. Scand., 1939, 83, $5-135$.

[25] Youssef E.F., Shanb A.A., Ameer M.A., Shanab M.E., Impact of body weight on shifting of foot pressure among adult subjects, Acta Bioeng. Biomech., 2020, 22, 131-137, DOI: 10.37190/ABB-01595-2020-02. 